\title{
Expression of LRIG1, a Negative Regulator of EGFR, Is Dynamically Altered during Different Stages of Gastric Carcinogenesis
}

Sungsook Yu, ${ }^{*}$ Mijeong Yang, ${ }^{*}$ Kyung-Min Lim, ${ }^{\dagger}$ Yejin Cho, ${ }^{*}$ Hyunji Kim, ${ }^{*}$ Keunwook Lee, ${ }^{\ddagger}$ Sang-Ho Jeong, ${ }^{\S}$ Robert J. Coffey, ${ }^{\mathbb{\top}||}$ James R. Goldenring, ${ }^{{ }^{* * *}}$ and Ki Taek Nam*

From the Severance Biomedical Science Institute, * Brain Korea 21 PLUS Project for Medical Science, Yonsei University College of Medicine, Seoul, Republic of Korea; the College of Pharmacy, ${ }^{\dagger}$ Ewha Womans University, Seoul, Republic of Korea; the Department of Biomedical Science, ${ }^{\ddagger}$ Hallym University, Chuncheon, Republic of Korea; the Department of Surgery, ${ }^{\S}$ Gyeongsang National University Changwon Hospital, Gyeongsang National University, Changwon, Republic of Korea; and the Epithelial Biology Center, " the Department of Medicine," and the Section of Surgical Science, ** Vanderbilt University School of Medicine, Nashville, Tennessee

\author{
Accepted for publication \\ August 14, 2018. \\ Address correspondence to $\mathrm{Ki}$ \\ Taek Nam, D.V.M., Ph.D., \\ Severance Biomedical Science \\ Institute, Yonsei University \\ College of Medicine, 50-1 \\ Yonsei-ro, Seodaemun-gu, \\ Seoul 03722, Republic of \\ Korea. E-mail: kitaek@yuhs.
}

\begin{abstract}
Leucine-rich repeats and immunoglobulin-like domains (LRIG)-1 is a transmembrane protein that antagonizes epidermal growth factor receptor signaling in epithelial tissues. LRIG1 is down-regulated in various epithelial cancers, including bladder, breast, and colorectal cancer, suggesting that it functions as a tumor suppressor. However, its role in gastric carcinogenesis is not well understood. Here, we investigated the changes in LRIG1 expression during the stages of gastric cancer. We used a DMP-777 -induced spasmolytic polypeptide-expressing metaplasia mouse model and a tissue array of human gastric cancer lesions. The effects of LRIG1 knockdown were also assessed using the human gastric cancer cell line SNU638 in a xenograft model. LRIG1 expression varied over the course of gastric carcinogenesis, increasing in spasmolytic polypeptide-expressing metaplasia lesions but disappearing in intestinal metaplasia and cancer lesions, and the increase was concurrent with the up-regulation of epidermal growth factor receptor. In addition, LRIG1 knockdown promoted the tumorigenic potential in vitro, which was manifested as increased proliferation, invasiveness, and migration as well as increased tumor size in vivo in the xenograft model. Furthermore, LRIG1 expression was determined to be a positive prognostic biomarker for the survival of gastric cancer patients. Collectively, our findings indicate that LRIG1 expression is closely related wto gastric carcinogenesis and may play a vital role as a tumor suppressor through the modulation of epidermal growth factor receptor activity. (Am J Pathol 2018, 188: 2912-2923; https://doi.org/10.1016/j.ajpath.2018.08.006)
\end{abstract}

Gastric cancer (GC), one of the most common cancers, ${ }^{1}$ occurs via structural changes and metaplasia of the gastric mucosa. ${ }^{2}$ Chronic Helicobacter pylori infection, a major cause of the disease, induces prominent inflammation and loss of parietal cells (or oxyntic atrophy), ${ }^{3}$ which precedes gastric preneoplasia as it plays an important role in gastric mucosa dedifferentiation. ${ }^{3,4}$ In humans, oxyntic atrophy can progress to two types of metaplasia: spasmolytic polypeptide-expressing metaplasia (SPEM) and intestinal metaplasia (IM). ${ }^{4}$ SPEM displays morphologic characteristics similar to those of the deep antral glands or Brunner glands, but gastrin-producing cells are not observed in
Supported by National Research Foundation (NRF; funded by the Korean government) Korea Mouse Phenotyping Project grants NRF2016M3A9D5A01952416 and 2013M3A9D5072551, The Ministry of Science, ICT and Future Planning grants NRF-2017R1A2B2009850 and NRF-2017M3A9F3041234, and Ministry of Science and ICT grant 2018R1A5A2025286; Yonsei University College of Medicine faculty research grant 6-2015-0163; the Yonsei University Brain Korea 21 PLUS Project for Medical Science (K.T.N.); and Ministry of Education, Science and Technology NRF Basic Science Research Program grant NRF2015R1C1A2A01053575 (S.Y.). J.R.G. is supported by US Department of Veterans Affairs Merit Review Award grant I01BX000930 and NIH grant RO1 DK071590.

S.Y., M.Y., and K.-M.L. contributed equally to this work.

Disclosures: None declared. 
SPEM. SPEM is characterized by the expression of specific markers such as mucin-6 and trefoil factor (TFF)-2. ${ }^{2-6}$ Preneoplastic IM is characterized by the presence of intestinal goblet cells in the stomach, which does not normally occur, ${ }^{2-4}$ and by the expression of intestinal markers mucin-2 and TFF3, as well as other intestinal brush border markers, including sucrose-isomaltase, villin, mucin13 , and fatty acid-binding protein.,

Several mouse SPEM models can be experimentally established using Helicobacter felis infection or by DMP-777 or L-635 treatment. Chronic H. felis infection results in parietal cell loss and the induction of SPEM with inflammatory responses. ${ }^{7}$ Treatment with L-635, which is structurally related to $\beta$-lactam, also causes prominent parietal cell loss and induces SPEM with inflammation. ${ }^{8}$ DMP-777 is a cell-permeable neutrophil elastase inhibitor, ${ }^{3}$ and its repeated administration induces oxyntic atrophy in mice. Acute destruction of parietal cells leads to SPEM without an inflammatory response. These regions can, however, revert to a normal state on DMP-777 withdrawal. ${ }^{8}$

Leucine-rich repeats and immunoglobulin-like domains protein (LRIG)-1, the encoding gene for which is located on chromosome $3 \mathrm{p} 14.3$, is a transmembrane protein with an extracellular domain containing 15 leucine-rich repeats and three immunoglobulin (Ig)-like domains. LRIG1 can interact with all four extracellular region binding protein B receptor family members and regulate receptor levels by enhancing ubiquitination and subsequent lysosomal degradation, independent of ligands. ${ }^{9-11}$ LRIG1 is also a marker of human epithelial stem cells in a quiescent nonproliferative state. ${ }^{12}$ Genetic ablation of LRIG1 results in increased proliferation associated with stem cell expansion in vitro and epithelial hyperproliferation in vivo. ${ }^{12,13}$ Recent lineage tracing suggests that LRIG1 predominantly marks noncycling, long-lived stem cells of the +4 quiescent intestinal stem cell niche in the crypt ${ }^{14}$; LRIG1 also marks progenitor cells in the stomach that are involved in restoring gastric cell mucosa after DMP-777-induced acute damage. ${ }^{15}$

Loss of Apc in LRIG1-positive cells leads to intestinal adenomas, increased extracellular region binding protein B-1 to -3 expression, and duodenal adenomas. ${ }^{14}$ Lrig1-null mice also exhibit enlarged intestines and crypt expansion, with elevated extracellular region binding protein $\mathrm{B}-1$ to -3 levels throughout the small intestine. ${ }^{14,16}$ LRIG1 expression is down-regulated in several cancers, including bladder, lung, renal, breast, and colorectal cancer. ${ }^{17-20}$ Notably, epidermal growth factor receptor (EGFR) activation is inversely related to LRIG1 expression, ${ }^{16}$ suggesting that LRIG1 plays the role of a tumor suppressor through maintaining intestinal epithelial homeostasis.

Although the roles of LRIG1 in colorectal carcinogenesis are well established, little is known about its association with GC. Here we investigated Lrig1 expression in the stomachs of normal and DMP-777-treated mice. To study the role of LRIG1 in human gastric carcinogenesis, its expression was analyzed in the lesions of GC patients. It was also determined whether LRIG1 contributes to tumorigenicity using a GC cell line, SNU638, in vitro and a xenograft model in vivo.

\section{Materials and Methods}

\section{In Situ Hybridization}

Nucleotides 3537 to 4295 of the LRIGI sequence (XM_017006134.2) were amplified using PCR with primers $5^{\prime}$-GGGTCGCTCTACCCCAGTA-3' (forward) and $5^{\prime}$-TTCCATCCTTCCCACCCCG-3' (reverse) to synthesize the cRNA probe. The data discussed in this publication have been deposited in NCBI's Gene Expression Omnibus ${ }^{21}$ (https://www.ncbi.nlm.nih.gov/geo; accession number GSE2361). The PCR products were then cloned into pGEM-T Easy Vector (Promega, Madison, WI) and subjected to restriction digestion, followed by labeling with digoxigenin (DIG) and in vitro transcription using the DIG-RNA labeling kit (Roche Diagnostics, Basel, Switzerland). Four-micrometer sections from paraformaldehyde-fixed, paraffin-embedded tissue samples were deparaffinized and rehydrated, and then incubated with $0.5 \%$ acetic anhydride solution to remove nonspecific binding. Hybridization was performed with DIG-labeled sense and antisense cRNA probes in $20 \times$ saline sodium citrate containing $50 \%$ formamide at $42^{\circ} \mathrm{C}$ overnight. Slides were then washed in saline sodium citrate solution at $50^{\circ} \mathrm{C}$ and incubated with an anti-DIG Fab antibody (Roche Diagnostics) conjugated to alkaline phosphatase or anti-DIG rhodamine antibody (Roche Diagnostics) at $4^{\circ} \mathrm{C}$ overnight. To detect alkaline phosphatase, nitro blue tetrazolium/5-bromo-4-chloro-3-indolyl phosphate (NBT/ BCIP; Roche Diagnostics) was added. Nuclei were counterstained with Nuclear Fast Red (Vector Laboratories, Burlingame, CA).

\section{IHC Analysis}

Paraffin-embedded tissue sections were deparaffinized with xylene and rehydrated with $100 \%, 95 \%$, and $70 \%$ ethanol. Using a pressure cooker, antigens were retrieved in target retrieval solution (Dako, Glostrup, Denmark). Endogenous peroxidase activity was blocked with $3 \% \mathrm{H}_{2} \mathrm{O}_{2}$, followed by protein blocking in protein block serum-free ready-to-use solution (Dako). Slides were treated with primary antibody diluted in solution (Dako) and incubated in a humidified chamber at $4^{\circ} \mathrm{C}$ overnight. After washing with phosphatebuffered saline (PBS), the samples were incubated with secondary rabbit IgG (Dako), detected with Dako Envision + System-horseradish peroxidase DAB (Dako), and counterstained with Mayer hematoxylin (SigmaAldrich, St. Louis, MO). Anti-LRIG1 antibody was purchased from Biorbyt (Cambridge, UK), and anti-EGFR 
and anti-phospho-EGFR (Tyr1068) antibodies from Abcam (Cambridge, UK).

\section{Dual Detection Using in Situ Hybridization and IHC}

To evaluate the expression of Lrig1 and Tff2, a marker of SPEM, in the mouse model of DMP-777-treated stomach, samples were hybridized with DIG-labeled Lrig1 and detected by treatment with NBT/BCIP; antibodies against Tff2 (Abcam) and anti-mouse horseradish peroxidase-conjugated IgM antibody (Zymed, San Francisco, CA) were then used, and signals were visualized using the Vectastain $\mathrm{ABC}$ reagent (Vector Laboratories) according to the manufacturer's instructions. To detect colocalization of Lrig1 RNA and Ki67, hybridization with DIG-labeled Lrig1, and anti-DIG IgG-rhodamine treatment (Roche Diagnostics) were performed. Next, anti-Ki67 antibody (Abcam) and Alexa Fluor 488-conjugated anti-rabbit secondary antibody (Life Technologies, Carlsbad, CA) were used; nuclei were stained with DAPI (Vector Laboratories).

\section{DMP-777 Preparation and Inoculation}

DMP-777, a gift from DuPont Pharmaceuticals (Wilmington, DE), was used at a concentration of $2 \%$ in $0.5 \%$ methylcellulose. C57BL/6 mice (6 weeks) were treated with DMP-777 by oral gavage $(350 \mathrm{mg} / \mathrm{kg})$ once a day for 7 and 14 days.

\section{RNA Extraction and Real-Time Quantitative PCR}

Total RNA was extracted from GC cells using TRIzol (Life Technologies). Next, $1 \mu \mathrm{g}$ of DNase-treated total RNA was used to synthesize cDNA using the ImProm-II Reverse Transcription System (Promega). cDNA was analyzed using Power SYBR Green Master Mix (Applied Biosystems, Waltham, MA) on a StepOnePlus Real-Time PCR System (Applied Biosystems), using the following primers: $5^{\prime}$ GTCACGGTCGCTGCTAACTC-3' (forward) and $5^{\prime}$ TGGAAGGTGAGGCCCTCTAT-3' (reverse) for LRIG1, $5^{\prime}$ ATGCCCGCATTAGCTCTTAG-3' (forward) and $5^{\prime}$ GCAACTTCCCAAAATGTGCC- $3^{\prime}$ (reverse) for EGFR, and $5^{\prime}$-AGAGCTACGAGCTGCCTGAC- $3^{\prime}$ (forward) and $5^{\prime}$ AGCACTGTGTTGGCGTACAG-3' (reverse) for actin.

\section{Western Blot Analysis}

Total protein was harvested from cells using radioimmunoprecipitation assay lysis buffer $[150 \mathrm{mmol} / \mathrm{L}$ sodium chloride, $1 \%$ NP-40 or Triton X-100, $0.5 \%$ sodium deoxycholate, $0.1 \%$ sodium dodecyl sulfate, and $50 \mathrm{mmol} / \mathrm{L}$ Tris ( $\mathrm{pH}$ 8.0)] supplemented with Halt phosphatase/protease inhibitor cocktail (Thermo Fisher Scientific, Rockford, IL). Samples were separated by SDS-PAGE, transferred to polyvinylidene difluoride membranes (Millipore, Billerica, MA), and blotted using the following antibodies:
anti-LRIG1 obtained from Biorbyt (Cambridge, UK); antiEGFR, anti-phospho-EGFR (Tyr1068), anti-N-cadherin, and anti-glyceraldehyde-3-phosphate dehydrogenase from Abcam; anti-E-cadherin from Santa Cruz Biotechnology (Santa Cruz, CA); and anti-vimentin, anti-zinc finger $\mathrm{E}$ box-binding homeobox $1 /$ T-cell factor 8 , and anti-zinc finger protein SNAI1 (SNAIL) from Cell Signaling Technologies (Danvers, MA). Antibodies were used with horseradish peroxidase-conjugated secondary antibodies (Life Technologies), followed by development using VisGlow plus chemiluminescent substrate (Visual Protein Biotechnology, Taiwan); signal intensities were quantified using ImageJ software version 1.5 (NIH, Bethesda, MD; http://imagej.nih.gov/ij). Data are representative of more than four independent experiments.

\section{Generation of LRIG1-Knockdown Cell Lines}

Two shRNA lentiviral vectors (in pGIPZ lentiviral shRNA vector) for LRIG1 (V2LHS_229246 and V2LHS_404471) were purchased from Open Biosystems (Huntsville, AL). Recombinant lentiviruses were produced by transfecting 293T cells with the packaging plasmids PMD2G and psPAX2 using the CalPhos Mammalian Transfection Kit (Clontech, Mountain View, CA) according to the manufacturer's protocol. LRIG1 knockdown was obtained by recombinant lentivirus infection. The GC cell line SNU638 was maintained in RPMI1640 (Sigma-Aldrich) medium with $10 \%$ fetal bovine serum and $1 \%$ penicillin/streptomycin at $37^{\circ} \mathrm{C}$ in a humidified atmosphere of $5 \% \mathrm{CO}_{2}$, followed by incubation with viral supernatant and polybrene $(10 \mu \mathrm{g} / \mathrm{mL})$; stable shRNA-expressing clones were selected using puromycin $(1 \mu \mathrm{g} / \mathrm{mL})$ for 3 days.

\section{Cell Viability Assays}

For evaluating proliferation, cells were seeded in 6-well culture dishes at $5 \times 10^{4}$ cells $/ 60-\mathrm{mm}$ dish. After the indicated time points, the cells were trypsinized, washed with PBS, and counted daily for 5 days using an automatic cell counter (EVE; Nanoentek, Seoul, Republic of Korea). Growth curves were plotted based on cell numbers. Data are representative of more than four independent experiments.

\section{Invasion and Migration Assays}

Cells transduced with $L R I G 1$ or shRNA against LRIGl were plated at $5 \times 10^{4}$ cells/well in 24-well Boyden chambers with $8-\mu \mathrm{m}$-pore polycarbonate membranes (Corning, Lowell, MA) according to the manufacturer's protocol. Cells were seeded in serum-free media and allowed to migrate for 24 hours toward the lower chamber containing media with $10 \%$ fetal bovine serum. The cells were then fixed with 5\% glutaraldehyde and stained with crystal violet (BD Biosciences, Franklin Lakes, NJ). For cell invasion, chambers were precoated with Matrigel (Corning). The 
migrated cells were imaged and counted from 10 microscopic fields using an Olympus microscope (Tokyo, Japan) with Topview software version 3.7 (SPECwise, West Palm Beach, FL) using a $\times 10$ objective. Results were analyzed using ImageJ software based on data from five independent experiments.

\section{Cell Cycle Analysis Using Flow Cytometry}

Cells were fixed with $2 \%$ paraformaldehyde, washed with PBS, treated with $0.1 \%$ Triton X-100/1\% BSA/PBS, and stained with FxCycle stains (Thermo Fisher Scientific). After staining, flow cytometric analysis was performed using a flow cytometer (BD Biosciences). Results were analyzed using FlowJo VX software version 10 (FlowJo LLC, Ashland, OR). Data are presented as the means \pm SEM from three independent trials.

\section{Xenograft Model of Human GC}

Male athymic nude mice were purchased from Taconic Biosciences (Rensselaer, NY). Animal studies were conducted according to protocols approved by the Institutional Animal Care and Use Committee of Yonsei University (Seoul, Republic of Korea). Cells were harvested using trypsin and washed twice with cold sterile PBS without calcium chloride or magnesium chloride (Sigma-Aldrich). The cells $\left(2 \times 10^{7}\right)$ were washed twice with cold sterile
$\mathrm{PBS}^{-1-}$ and mixed at a ratio of 50:50 (v/v) with Matrigel (Corning). Cells were injected s.c. into the dorsal flank of 7-week-old male athymic nude mice to establish primary tumors. Tumor volumes were measured using calipers and calculated as follows: $V=\pi \times(W \times H \times D) / 6$ (W, shortest diameter; $H$, longest diameter; $D$, depth). Each mouse was analyzed using IVIS (Caliper Life Sciences, Hopkinton, MA) at an excitation wavelength of $710 \mathrm{~nm}$ and an emission wavelength of $760 \mathrm{~nm}$. Animals were then sacrificed, and selected tissues were processed for histology.

\section{Statistical Analysis}

Statistical analyses were performed using JMP software version 4 (SAS, Cary, NC). The $t$-test was used to analyze mean differences. Data are expressed as means \pm SEM.

\section{Results}

\section{Lrig1 Expression in Normal Mouse Gastrointestinal Epithelium}

The expression and localization of Lrigl was examined in the gastric mucosa and intestinal epithelium of normal mice using in situ hybridization (Figure 1). Lrig1-positive cells were observed in the stomach at the isthmus region of the fundus, and in the antral glands at the base of the antrum (Figure 1A), where gastric stem cells reside.
A

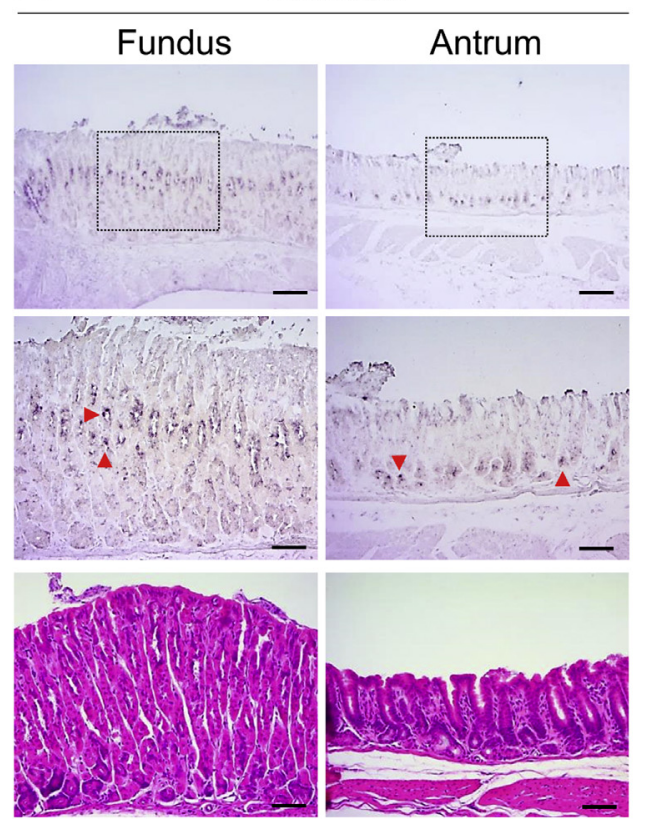

B

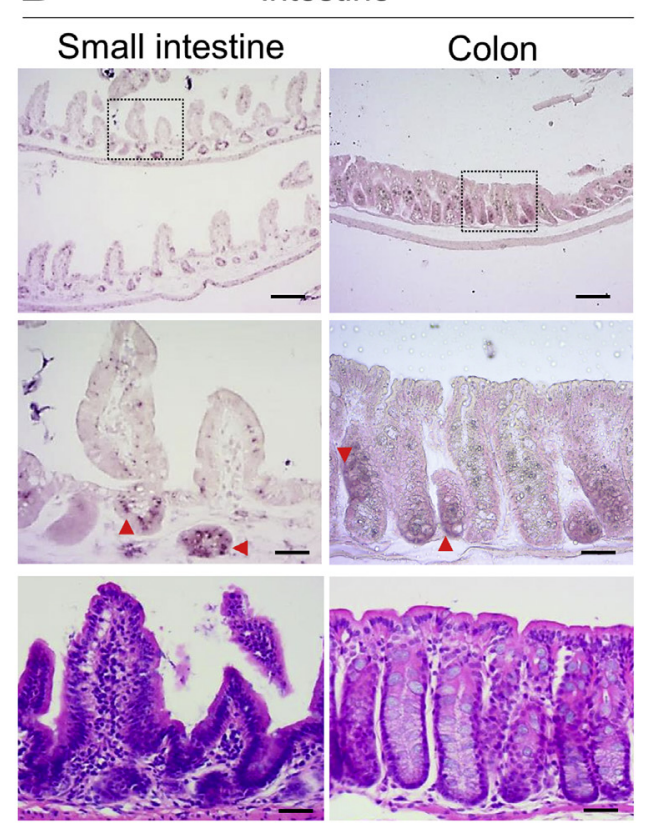

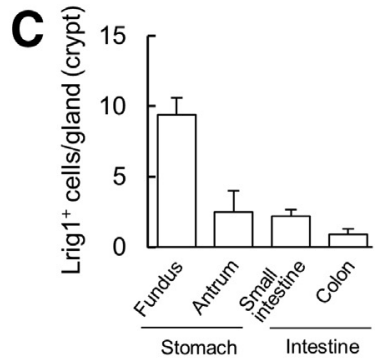

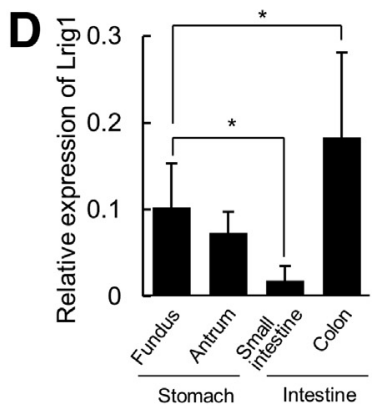

Figure 1 Lrig1 expression in the gastrointestinal epithelium of normal mouse determined using in situ hybridization (ISH). A and B: Localization of Lrig1 expression was detected with ISH in the fundus and antrum of the mouse stomach (A) and intestine (B). Boxed areas are shown at a higher magnification in the middle row. Arrowheads indicate Lrig1-positive cells. Bottom images are hematoxylin and eosin stained. C: Leucine-rich repeats and immunoglobulin-like domains protein (Lrig)-1-positive cells were counted from 10 microscopic fields. Statistical analyses were performed using JMP software version 4. D: Relative Lrig1 expression was measured by real-time quantitative PCR. Data are expressed as means \pm SEM. $n=4$. ${ }^{*} P<0.05$. Scale bars: $500 \mu \mathrm{m}(\mathbf{A}$, top row); $100 \mu \mathrm{m}$ (A, middle row and bottom row); $200 \mu \mathrm{m}$ (B, top row); $50 \mu \mathrm{m}$ (B, middle row and bottom row). 
Lrig1-positive cells were also detected at the bottom of the crypt in the small intestine and the colon (Figure 1B), a stem cell-rich zone. Relative levels of Lrig 1 RNA expression in the gastrointestinal epithelium were observed in the following order: colon $>$ stomach (fundus $\cong$ antrum) $>$ small intestine (Figure 1, C and D).

\section{Increased Lrig1 Expression in Metaplastic Mouse Stomach}

It was then examined whether metaplasia could induce changes in Lrigl expression in the gastric mucosa in vivo. For this, in situ hybridization was performed in the stomachs of a DMP-777-treated mouse SPEM model at days 0,7 , and 14 after administration, and after 14 days of recovery without treatment. At day 0 , relatively few Lrig1-positive cells were observed in the isthmus region of the fundus and in the antral gland of the gastric mucosa (Figure 2A). After 7 days of DMP-777 treatment, the number of Lrigl-positive cells increased prominently along the glands in the metaplastic region of the fundus, and further increases were detected until day 14. Interestingly, when DMP-777 treatment was withdrawn for 14 days to allow the gastric mucosa to recover, Lrig 1 expression reverted to normal levels. However, only minimal changes were noted in the antrum compared to the fundus after 7 days of DMP-777 administration (Figure 2A).

To identify whether Lrigl expression was enhanced in the metaplastic lesion, localization of Lrig 1 and Tff2, a marker of SPEM, was determined using in situ hybridization and immunohistochemistry analysis. Tff2 and Lrig1 double-positive cells appeared along the glands of DMP-777-treated mice (Figure 2B). Next, to examine whether Lrig1 expression was increased in gastric stem cells with metaplasia, in situ hybridization for Lrigl and subsequent staining for $\mathrm{Ki}-67$, a proliferative cell marker, were performed and revealed that the Lrig1-positive cells were proliferative (Figure 2C).
A

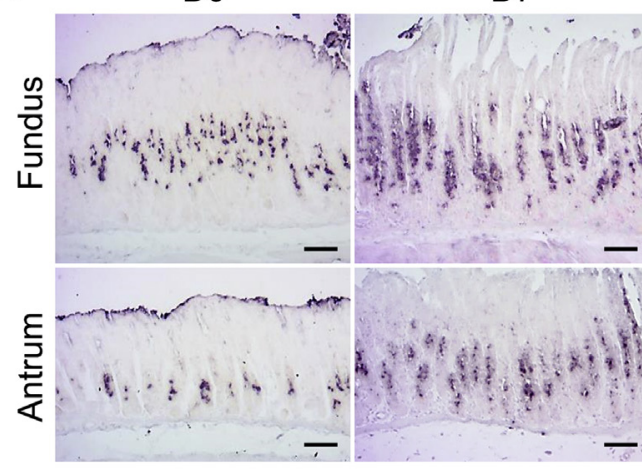

B

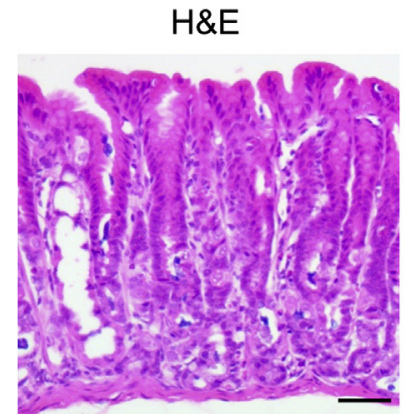

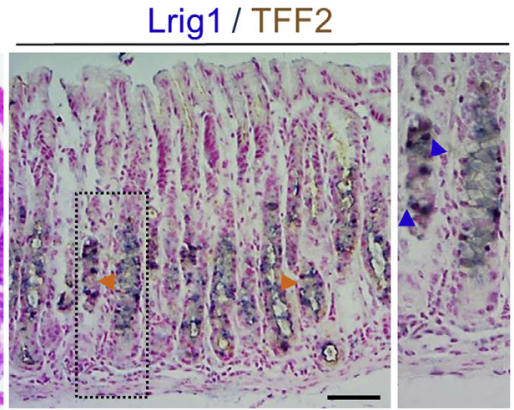

D14
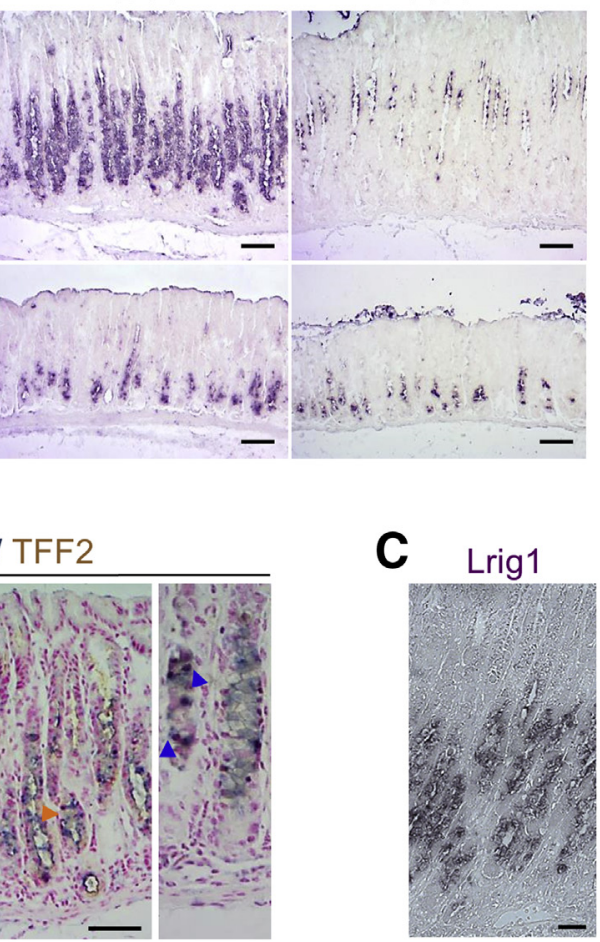
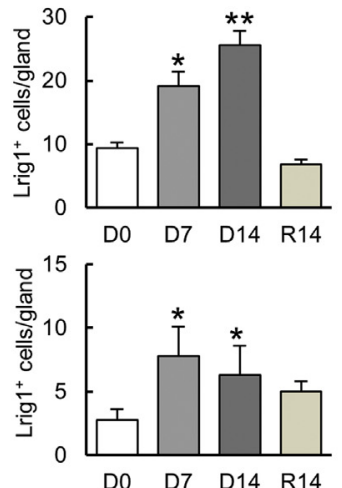

Ki-67

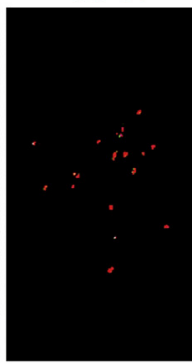

Lrig1/Ki-67

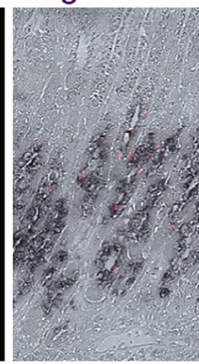

Figure 2 Lrig1 expression in a mouse model of DMP-777-induced spasmolytic polypeptide-expressing metaplasia (SPEM). SPEM was induced in mice with DMP-777 treatment. A: Lrig1 expression was detected using in situ hybridization (ISH), with anti-digoxigenin (DIG) Fab antibody conjugated with alkaline phosphatase. Lrig1-positive cells from 10 microscopic fields were counted. Statistical analyses were performed using JMP software version 4. B: Leucine-rich repeats and immunoglobulin-like domains protein (Lrig)-1 expression was detected in metaplasias from mice treated with DMP-777 for 14 days. ISH and immunohistochemistry analysis were performed using DIG-labeled Lrig1 probe (blue) and anti-trefoil factor (Tff)-2 antibody (brown), a marker of spasmolytic polypeptide-expressing metaplasia. Boxed area is shown at higher magnification to the right. Orange arrowheads indicate Tff2-positive cells; blue arrowheads, Lrig1-positive cells. C: Lrig1-positive cells show proliferative ability. ISH for Lrig1 was performed using detection of nitro blue tetrazolium/5bromo-4-chloro-3-indolyl phosphate and antibody against Ki-67 (red). Sections include fundic mucosa from mice treated with DMP-777 for 14 days. Data are expressed as means \pm SEM. ${ }^{*} P<0.05$ and ${ }^{*} P P<0.01$ versus DO. Scale bars $=100 \mu \mathrm{m}$. D0, untreated mouse; D7 or D14, mouse treated with DMP-777 for 7 or 14 days, respectively; and R14, mouse treated with DMP-777 for 14 days and allowed to recover for 14 days. H\&E, hematoxylin and eosin. 


\section{LRIG1 Expression during Human Gastric Carcinogenesis}

To evaluate changes in LRIGl expression during gastric carcinogenesis, LRIG1 RNA expression was determined in GC-patient specimens from normal, SPEM, IM, and cancerous regions; the expression patterns were similar to those observed in the mouse model (Figure 3). In normal human gastric tissue, LRIG1-positive cells were detected at the isthmus of the gastric mucosa (Figure 3, A and B). However, LRIG1 expression was dynamically altered during GC development. Compared to that in normal gastric tissue, LRIGl-positive cells were significantly increased in the SPEM region (Figure 3, C, D, and G). However, LRIGI expression was minimal in the IM region (Figure 3, C and D) and became nearly undetectable in cancerous tissue (Figure 3, E, F, and G). As in in situ hybridization, LRIG1 immunoreactivity in immunohistochemistry analysis (Figure 4) was strong in the SPEM region (Figure 4A), weak in the IM region (Figure 4C), and absent in the cancerous lesions (Figure 4E). EGFR expression was detected in a trend opposite to that of
$L R I G 1$, supporting the role of LRIG1 in EGFR regulation. In the SPEM region, EGFR expression was weak (Figure 4, B and D), whereas that in IM was strong (Figure 4D). Interestingly, although EGFR-positive foveolae with an EGFR-negative base were difficult to observe in the glands of SPEM region, those of EGFRpositive foveolae with an EGFR-negative base existed at a level of $5.35 \% \pm 3.5 \%$ in the IM region. In the cancerous lesions, EGFR immunoreactivity was very strong (Figure 4F), corroborating the negative regulation of EGFR by LRIG1 (Figure 4G).

\section{Effects of LRIG1 Knockdown on Gastric Carcinogenesis}

To further clarify the role of LRIG1 in gastric carcinogenesis, the tumorigenic potential of LRIG1 was examined with respect to the epithelial-to-mesenchymal transition (EMT) using human gastric tumor cell line SNU638. EMT is key to motility, invasion, metastasis, tumor-initiating capacity, and resistance to chemotherapy in tumor cells. ${ }^{22}$ Using SNU638 clones transfected with pGIPZ lentiviral vector (control
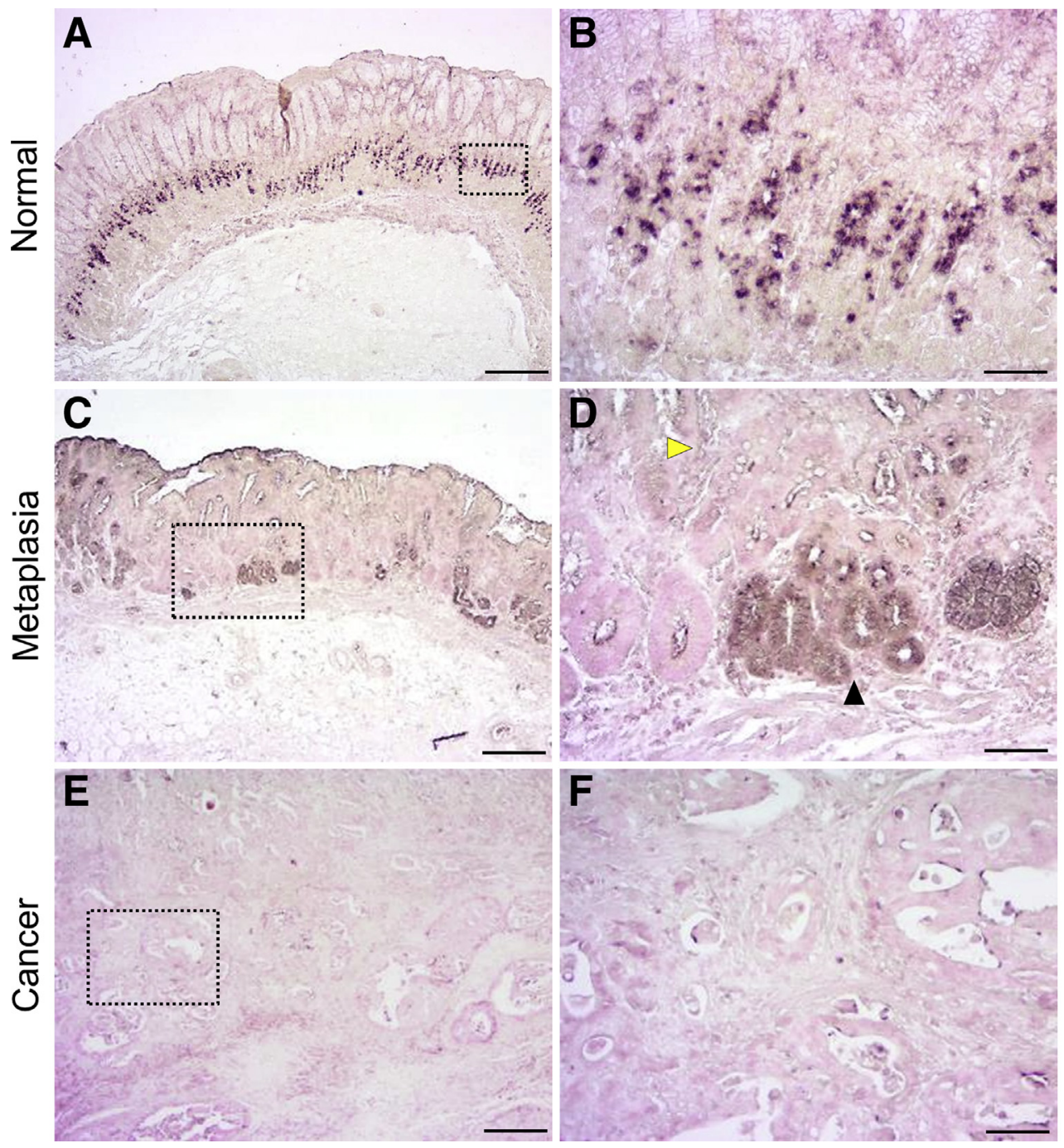

Figure 3 LRIG1 expression in human gastric normal, preneoplastic, and cancer tissues determined with in situ hybridization. A-F: LRIG1 expression was detected in human gastric normal (A and $\mathbf{B}$ ), metaplastic ( $\mathbf{C}$ and $\mathbf{D})$, and cancer ( $\mathbf{E}$ and $\mathbf{F}$ ) tissues, using digoxigenin-labeled LRIG1 probe. LRIG1 expression is significantly increased in a spasmolytic polypeptide-expressing metaplastic region (black arrowhead), but not in intestinal metaplastic region (yellow arrowhead). Nuclei were stained with Nuclear Fast Red. Boxed areas are shown at higher magnification in the right column. G: LRIG1-positive cells from 10 microscopic fields were counted. Samples from six patients were used in these experiments. Data are expressed as means \pm SEM. $* * P<0.01$ and $* * * P<0.001$. Scale bars: $500 \mu \mathrm{m}$ (A); $50 \mu \mathrm{m}$ (B, D, and F); $200 \mu \mathrm{m}$ (C and $\mathbf{E})$.

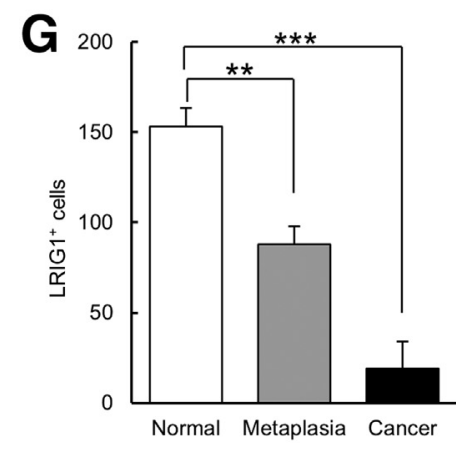



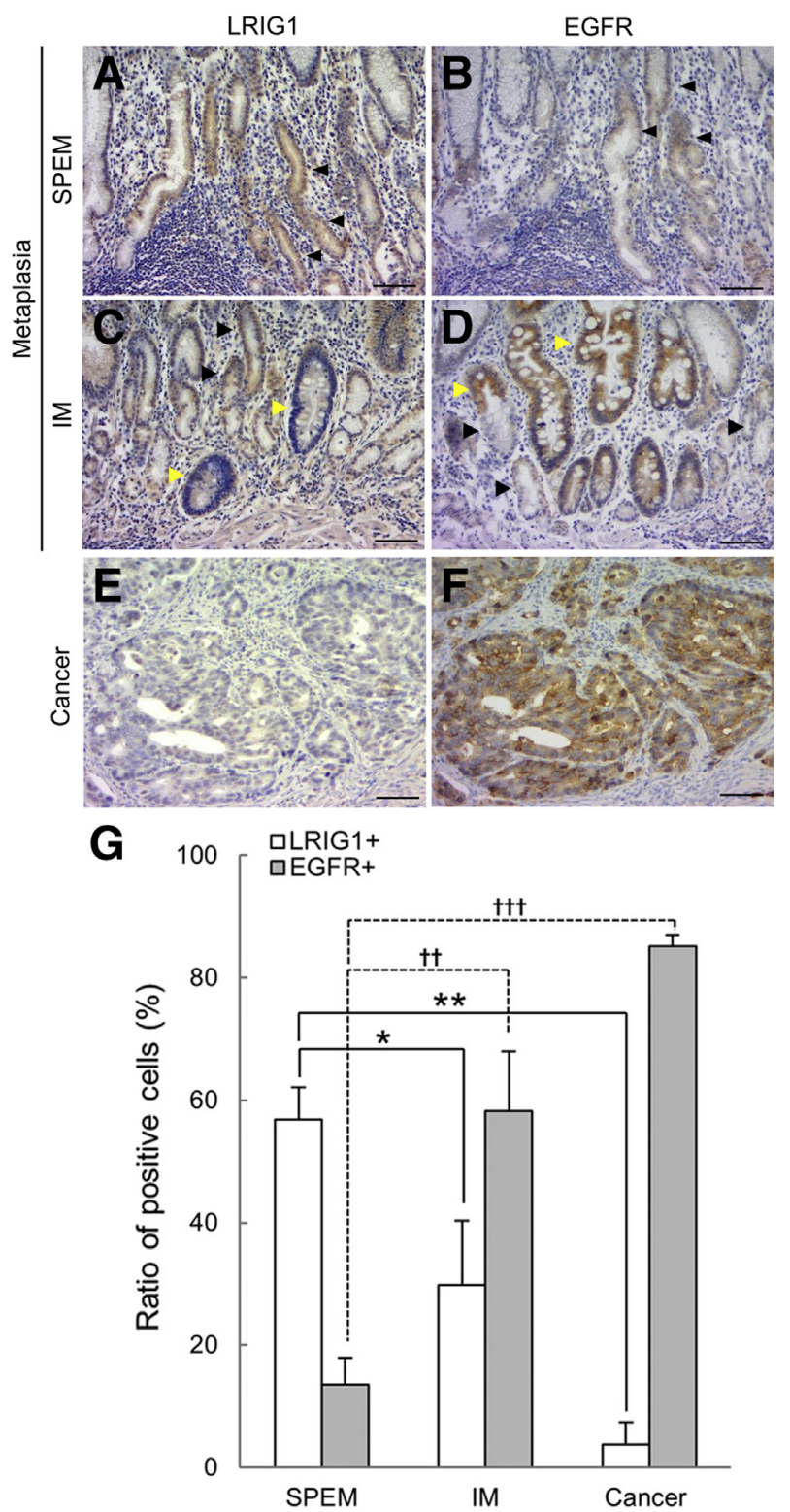

Figure 4 Immunohistochemistry (IHC) analysis of leucine-rich repeats and immunoglobulin-like domains protein (LRIG)-1 and epidermal growth factor receptor (EGFR) expression in human gastric preneoplastic and cancer tissues. LRIG1 and EGFR were detected in human gastric preneoplastic and cancer tissues using IHC with indicated antibodies. A and B: In a spasmolytic polypeptide-expressing metaplastic (SPEM) region (A), LRIG1 was expressed strongly, whereas EGFR expression was low along SPEM glands (arrowheads). C-E: Decreased LRIG1 expression (C and D; yellow arrowheads) and low EGFR gland expression ( $\mathbf{C}$ and $\mathbf{D}$; black arrowheads) are observed in the intestinal metaplastic (IM) region, and LRIG1 is absent in cancer tissues (E). D and F: Strong EGFR expression in the IM region (D, yellow arrowheads) and in cancer lesions $(\mathbf{F})$ is shown. Samples from five patients were used in these experiments G: LRIG1- or EGFR-positive cells were counted from 10 microscopic fields. Statistical analyses were performed using JMP software version 4. Data are expressed as means \pm SEM. ${ }^{*} P<0.05$ and ${ }^{* *} P<0.01 ;{ }^{\dagger \dagger} P<0.01$ and ${ }^{\dagger \dagger} P<0.001$. Scale bars $=100 \mu \mathrm{m}$.

virus), pGIPZ-sh46, or pGIPZ-sh71 viruses, the effect of LRIG1 depletion was examined by Western blot analysis (Figure 5A). LRIG1 depletion induced up-regulation of EGFR and phospho-EGFR and altered the expression of EMT- associated proteins. In addition, loss of LRIG1 up-regulated $\mathrm{N}$-cadherin, a mesenchymal marker, as well as the transcription factors zinc finger $\mathrm{E}$ box-binding homeobox 1 and SNAIL, which are master regulators of EMT. The expression of vimentin, representing cytoskeletal changes that anchor focal adhesion complexes to promote cell migration, was also obviously altered. Conversely, the expression of E-cadherin and occludin, which are epithelial markers, was decreased in the LRIG1-knockdown cells (Figure 5A).

Next, it was investigated whether LRIG1 depletion affected the proliferation and migration of SNU638 cells. LRIG1 knockdown increased cell proliferation after 3 days compared with the pGIPZ-transduced cells (Figure 5B). Moreover, LRIG1 depletion (sh46 and sh71) increased the proportion of cells in the $\mathrm{G}_{2} / \mathrm{M}$ phase (indicating growing cells) from $19.6 \% \pm 1.53 \%$ to $29.0 \% \pm 2.80 \%$ and $27.1 \% \pm 3.01 \%$, respectively (Figure $5 \mathrm{C}$ ). Cells in the ratelimiting step of the cell cycle $\left(\mathrm{G}_{0} / \mathrm{G}_{1}\right)$ decreased from $52.6 \% \pm 3.02 \%$ to $44.6 \% \pm 4.00 \%$ and $39.3 \% \pm 1.98 \%$, respectively. Furthermore, LRIG1-depleted cells showed enhanced invasion and migration, which are phenotypic changes distinctive of EMT (Figure 5, D and E).

\section{LRIG1 Down-Regulation Increases Tumor Growth in Vivo}

Finally, the effects of LRIG1 depletion were examined on the growth of GC cells in vivo. SNU638 clones transfected with either pGIPZ (control vector) or shRNA (sh46 and sh71) were used in a mouse xenograft model (Figure 6, A and B). Tumor volume was monitored over 8 weeks (Figure 6A). Whereas untreated SNU638 and pGIPZtransduced SNU638 failed to maintain the initial tumor volume, SNU638 clones with LRIG1 knockdown (sh46 and sh71) made significantly larger tumor volumes after 4 weeks of injection. Typical tumor morphology, such as active mitosis and a high nucleus/cytoplasm ratio, was observed in the tissues injected with untreated or pGIPZ-transducedSNU638 clones. In contrast, extensive necrosis, characterized by pyknosis and obliteration of cell morphology, was observed after LRIG1 knockdown (sh46 and sh71) (Figure 6C). Moreover, enhanced activation of EGFR [phosphorylated (p)-EGFR] after LRIG1 depletion was observed (Figure 6C).

\section{LRIG1 Expression Is Related to GC Patient Survival}

Next, to examine whether LRIG1 expression in the stomach has prognostic value with regard to overall survival and relapse-free survival of GC patients, Kaplan-Meier plots were generated using mRNA data sets of $1065 \mathrm{GC}$ patients from Gene Expression Omnibus ${ }^{21}$ (Affymetrix microarrays only; https://www.ncbi.nlm.nih.gov/geo; accession numbers GSE14210, GSE15459, GSE22377, GSE29272, and GSE51105) with an online plotter (http://kmplot.com/ analysis/index.php? $p=$ service \& cancer $=$ gastric ${ }^{23}$ widely 


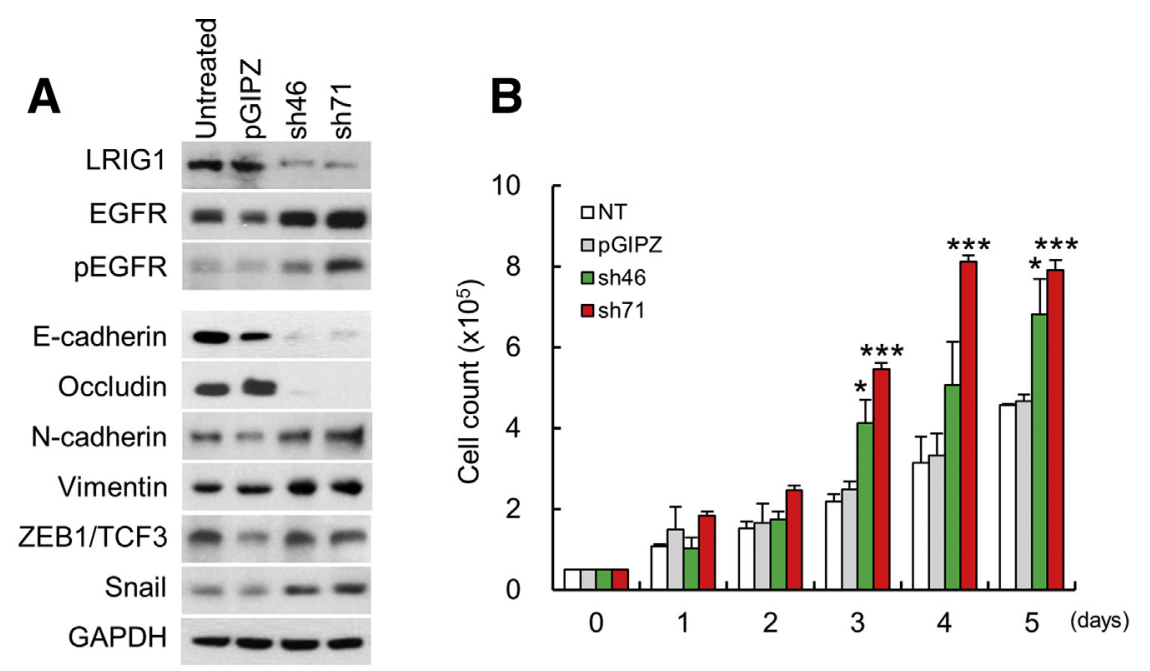

C

D
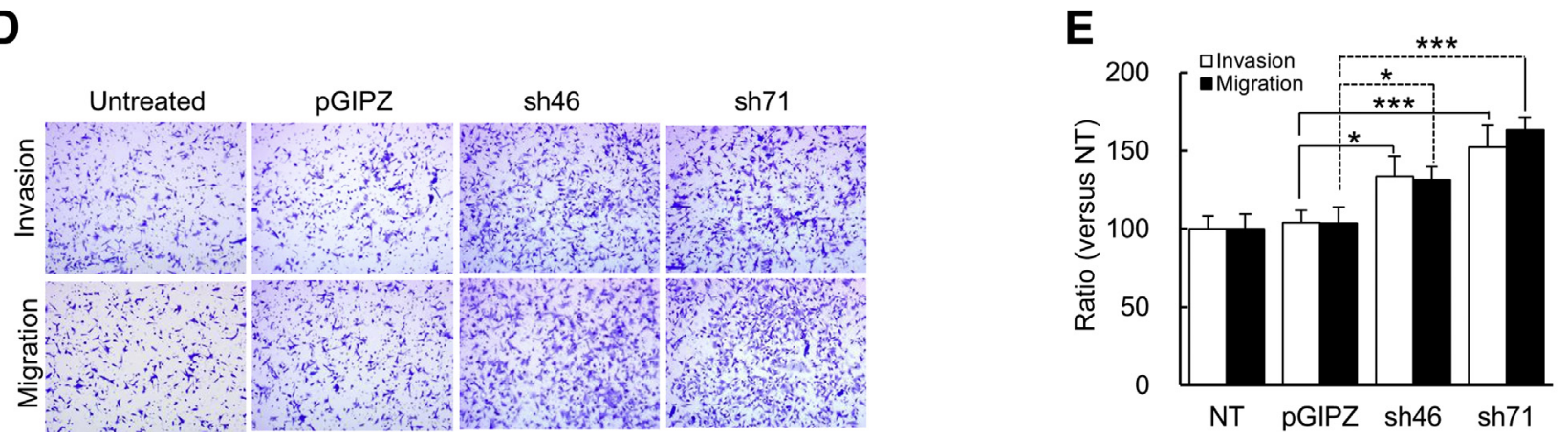

Figure 5 Effects of leucine-rich repeats and immunoglobulin-like domains protein (LRIG)-1 knockdown on tumorigenesis in the SNU638 gastric cancer cell line. A: LRIG1 was depleted using pGIPZ-based shRNA (sh46 and sh71). Levels of LRIG1, epidermal growth factor receptor (EGFR), phospho-EGFR, and epithelial to mesenchymal markers [E-cadherin, occludin, N-cadherin, vimentin, zinc finger E box-binding homeobox (ZEB)-1/T-cell factor (TCF)-3, and anti-zinc finger protein SNAI1 (SNAIL)] were detected by Western blot analysis using glyceraldehyde-3-phosphate dehydrogenase (GAPDH) as a loading control. B: Cell proliferation was assessed with cell counting over 5 days. C: Cell cycle was examined with flow cytometry. D: Representative image of invasion and migration analyzed using Boyden chambers. E: Quantitative-analytic data from four independent experiments. Data are expressed as means \pm SEM. $n=3$ (C); $n=4$ (A, B, and $\mathbf{D})$. ${ }^{*} P<0.05$ and ${ }^{* * *} P<0.001$ versus pGIPZ control. NT, non-treated.

used to examine the association between specific molecules and cancer progression. ${ }^{24}$ The Kaplan-Meier graphs showed that relapse-free survival in $593 \mathrm{GC}$ patients (Figure 6D), monitored through 150 months, separated into high or low based on LRIG1 mRNA expression levels. These patients showed relative LRIG1 expression levels from 64 to 5084 (arbitrary units) and were classified as low or high based on a cutoff of 1036, the median value. The survival numbers at the 50-month follow-up point were 49 of 295 versus 91 of 298 in the low and high groups, respectively. The difference in survival ratios was maintained to the 100-month followup point (15/295 vs 26/298). Higher LRIG1 expression was verified as a positive prognostic biomarker for increased relapse-free survival in GC patients [hazard ratio $=0.73(0$. 6 to 0.88$), \log \operatorname{rank} P=0.0012$ ].

\section{Discussion}

We demonstrated a negative relationship between LRIG1 expression and gastric carcinogenesis. LRIG1 expression was also negatively correlated with EGFR levels and EGFR activation. LRIG1 expression was significantly increased in SPEM, but low in IM and absent in GC lesions. LRIG1 knockdown promoted growth, metastasis, invasion, and EMT of SNU638 GC cells, and its expression was positively and significantly associated with relapse-free survival in GC patients. These findings indicated that LRIG1 might function as a tumor suppressor in gastric carcinogenesis.

Although multiple studies have suggested the role of LRIG1 as a tumor suppressor in various epithelial cancers, little is known about the function of LRIG1 in stomach and gastric carcinogenesis. In the normal mouse stomach, Lrig1 was detected at the isthmus of the fundus and antral glands, where stem cells are highly enriched ${ }^{25}$; these results suggested a role for LRIG1 in cell proliferation. Consistent with this finding, a recent study reported that LRIG1 marks stem cells that regenerate acutely in the damaged gastric mucosa. ${ }^{15}$ In support of this report, colocalization of Lrig1 and $\mathrm{Ki}-67$ was detected in DMP-777-treated mice. 

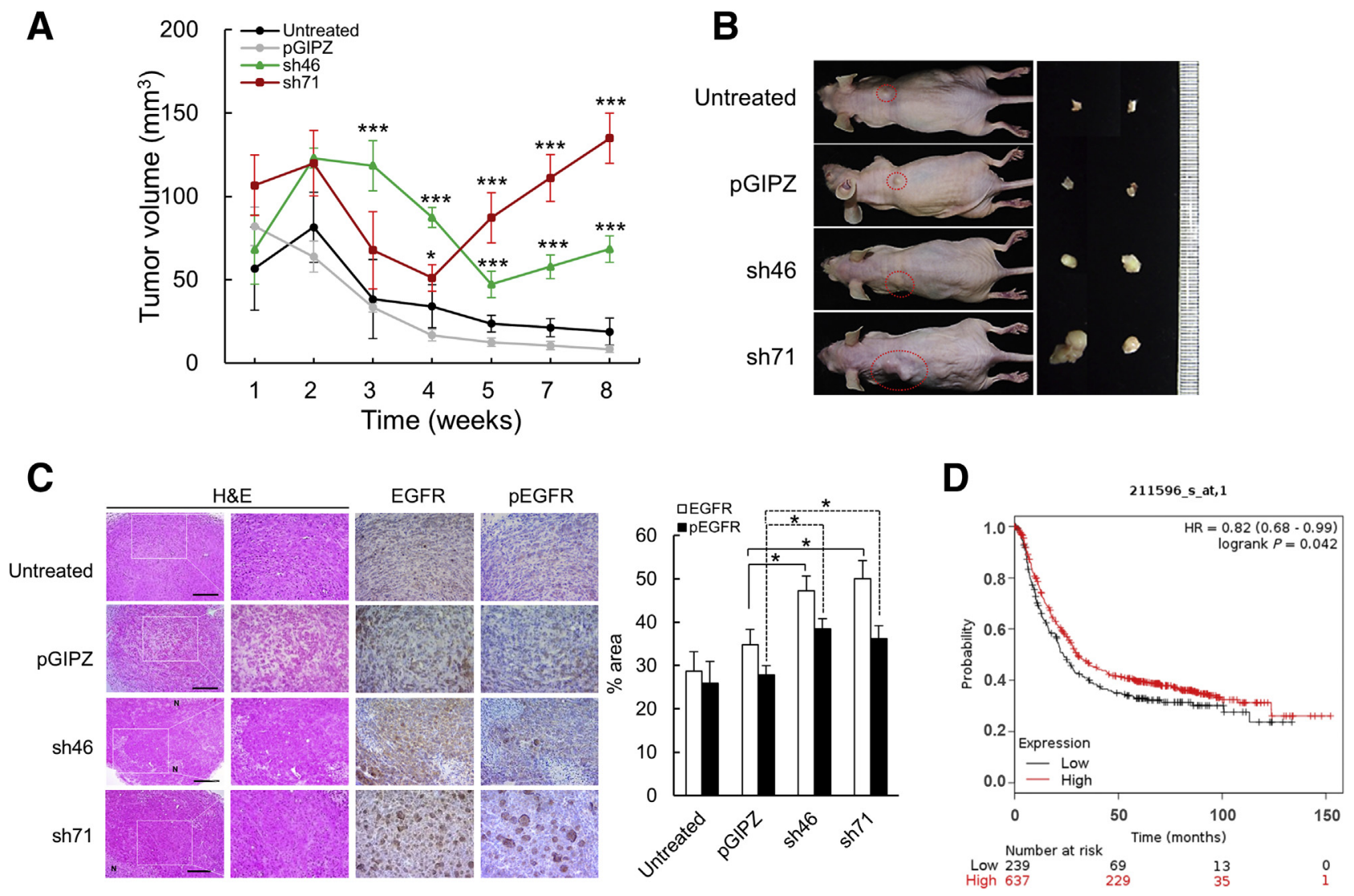

Figure 6 Effects of leucine-rich repeats and immunoglobulin-like domains protein (LRIG)-1 knockdown on SNU638 xenografts and Kaplan-Meier survival curves of relapse-free survival (RFS) of gastric cancer patients with low or high LRIG1 mRNA expression. SNU638 cells, with or without vector transfection, were injected s.c. into the dorsal flank of 7-week-old male athymic nude mice to establish primary tumors. A: Tumor volumes were measured using calipers. B: Extent of tumor growth was evaluated in vivo. Dotted circles represent tumor mass. C: Histopathologic analysis of gastric tumor masses from mouse xenograft model. Hematoxylin and eosin (H\&E) staining was performed to investigate epidermal growth factor receptor (EGFR) and phospho (p)-EGFR (Tyr1068) expression in tumor tissues from xenograft mice. Boxed areas are shown at higher magnification to the right. EGFR- and pEGFR-positive cells were counted from five microscopic fields. Statistical analyses were performed using JMP software version 4. D: Kaplan-Meier curves show RFS in gastric cancer patients, monitored for 150 months, based on high or low tumor LRIG1 mRNA expression levels. Red, patients with expression levels above the median; black, patients with expression levels below the median. Patients with low expression levels had a lower probability of RFS over time. Data are expressed as means \pm SEM. $n=4$ mice per treatment. ${ }^{*} P<0.05$ and ${ }^{* *} * P<0.001$ versus pGIPZ control. Scale bars $=100 \mu \mathrm{m}$.

Notably, Lrig1-positive cells increased after DMP-777 treatment in a time-dependent manner, and Lrig1 was coexpressed with Tff2, a SPEM marker, confirming its role in the development of these lesions. This finding is also consistent with findings from a previous report indicating that loss of Lrig1 results in duodenal adenomas with gastric metaplasia. ${ }^{26}$ Interestingly, LRIG1 expression was increased in human SPEM lesions, but was lost in IM and GC lesions, all of which suggests that it plays a dynamic role in human gastric carcinogenesis.

Gastric carcinogenesis takes place sequentially, beginning with chronic gastritis and progressing to gastric atrophy, IM, and dysplasia. ${ }^{27-29}$ Within the stomach, SPEM and IM are the predominant origins of metaplasia. Acute injury or ulceration leads to parietal cell loss, which often precedes the progression of SPEM. SPEM then proceeds to IM in the presence of chronic inflammation. Although SPEM and IM both exist in the human stomach with atrophic gastritis secondary to $H$. pylori infection, ${ }^{30,31}$ there are limited mouse models with IM. In our previous studies, amphiregulin-deficient mice spontaneously developed SPEM within the first 6 months. In a subset of those mice, goblet-cell IM advanced, indicating the existence of compound glands containing both SPEM and IM. ${ }^{32}$ Although clarification of the connection between IM and SPEM using murine studies has been insufficient, studies in Mongolian gerbils have been sources of additional insight. On H. pylori infection, both SPEM and IM can be seen in the fundus of Mongolian gerbils. ${ }^{33-35}$ IM developed in preexisting SPEM glands, and mixed glands expressing both SPEM and IM were identified in the gerbils. ${ }^{33}$ These studies support the hypothesis of IM development from preexisting SPEM. Furthermore, in our previous studies, the examination of human gastric sections containing regions of both SPEM and IM led to the identification of regions with compound glands, in which SPEM cells were observed in the deep portions with IM lineages in the luminal regions. ${ }^{31}$ This observation serves as convincing support that SPEM 
gives rise to IM. The elevated proliferation in IM adjacent to SPEM may indicate a transition or secondary differentiation of SPEM into IM. ${ }^{31}$

Lrig1-positive cells in the isthmus region, which were increased in SPEM after treatment with DMP-777, are thought to be correlated with the repair response to gastric epithelial cell damage. To repair gastric mucosal injury, reprogramming of chief cells into a pyloric-type metaplasiaSPEM occurs, which often advances into further stages of metaplasia, including IM under certain conditions such as chronic inflammation or in the presence of activated macrophages. ${ }^{36-39}$ Furthermore, increasing evidence indicates that SPEM likely represents an initial metaplastic response to gastric and chronic injury, and inflammation may lead to evolution into goblet-cell IM.,30 Thus, although pyloric-type metaplasias including SPEM in the stomach are initially reparative, their persistence in the setting of chronic inflammation can lead to deleterious neoplastic scenarios. ${ }^{40}$ Lrig was associated with quiescent multipotent stem cells in the intestine, such as in the case of SPEM, demonstrating its important roles in the homeostasis and regulation of self-renewal. ${ }^{14}$ In SPEM, genetic alteration can occur by mutations of progenitor cells or stem cells accumulated during repeated regeneration of the epithelial lineage; eventually, the expansion of cells with modified genetic information could overwhelm the homeostasis and regulation, ultimately leading to carcinogenesis. Notably, LRIG1 expression can distinguish SPEM from IM, suggesting that LRIGl acts as a pivotal genetic modulator of SPEM/IM progression by regulating proliferation, although further studies are needed to verify this hypothesis.

LRIG1 is a negative regulator of EGFR, ${ }^{10,11}$ and its role as a tumor suppressor has been suggested ${ }^{14}$; LRIG1 is proposed to be a key player in determining human cancer malignancy. ${ }^{41}$ Moreover, LRIG1 and EGFR are thought to interact via ectodomains. Goldoni et $\mathrm{al}^{42}$ showed that a soluble ectodomain of LRIG1 containing 15 leucine-rich repeat domains modulated EGFR signaling and growthpromoting activity. ${ }^{9}$ This finding was further supported in colon cancer, where LRIGl knockdown resulted in EGFR/ AKT activation as well as downstream signaling pathways leading to apoptosis evasion. ${ }^{43}$ Loss of LRIG1 expression has been confirmed in human GC lesions, whereas EGFR expression is significantly increased, which indicates that LRIG1 down-regulation might accelerate carcinogenesis through EGFR activation. Recently, Zhou et $\mathrm{al}^{44}$ reported that EGFR down-regulation through LRIGl inhibition by miR-20a affected apoptosis in the GC cell line SGC7901 with respect to the mitogen-activated protein kinase/ERK and phosphoinositide 3-kinase/AKT pathways, affecting the multidrug-resistance process. A similar study reported that the regulation of LRIG1 expression by miR-19a affected glioma cell growth. ${ }^{45}$ The results from those reports coincide with our findings, corroborating our conclusion regarding the role of LRIG1 attenuation in the setting of EGFR activation. Furthermore, through showing dynamic changes in EGFR in SPEM, IM, and GC lesions and the LRIGIknockdown GC cell line, which were inversely related to LRIG1 expression and positively linked with tumorigenesis, we believe that an important line of evidence for the interaction of LRIG1 and EGFR in tumorigenesis was provided.

The in vivo relevance of these results was supported by the findings from our experiments using a xenograft model. As with other studies in which a xenograft generated from SNU638 produced small-volume tumors, ${ }^{46,47}$ pGIPZtransduced SNU638 produced small-volume tumors. Nonetheless, LRIG1 knockdown in SNU638 led to increased tumor growth, which indicated the potential role of LRIG1 as a tumor suppressor in gastric carcinogenesis. In the early stage of tumor metastasis, the epithelial cancer cell phenotype changes to the mesenchymal phenotype by EMT. ${ }^{48,49}$ The analysis of the metastatic effect of LRIG1 showed that LRIG1 loss induced a mesenchymal phenotype indicated by up-regulation of mesenchymal cell markers, $\mathrm{N}$-cadherin, vimentin, and SNAIL as well as increased migration and invasion of GC cells. Thus, these data indicate that LRIG1 acts as a tumor suppressor in GC cells by regulating oncogenic behaviors. These results were further corroborated by patient-survival analysis, which showed that GC patients with low LRIG1 expression exhibited significantly lower relapse-free survival. Thus, these data suggest that LRIG1 functionally limits metastasis progression, acting as a barrier to EMT.

In conclusion, these findings indicate that LRIG1 is expressed in the stem cell-enriched isthmus of the fundus and antral glands of the antrum in the stomach. It was also found that LRIG1 expression is dynamically altered during metaplasia of the gastric mucosa and gastric carcinogenesis, and thus it appears to play the role of a tumor suppressor. Most importantly, it was demonstrated that LRIG1 was lost in IM, a precancerous lesion of adenocarcinoma and GC, which coincided with EGFR up-regulation. Conversely, it was shown that in SPEM, the very early stage of metaplasia, LRIG1 expression was up-regulated and EGFR was downregulated, which supports a dynamic inverse relationship between LRIG1 and EGFR during gastric tumorigenesis. Finally, Lrig1 down-regulation led to EGFR activation and EMT, which resulted in accelerated tumor growth, in vivo. These results provide an important clue regarding the key role of LRIG1 as a tumor suppressor in the stomach.

\section{Acknowledgment}

We thank DuPont Pharmaceuticals for providing the DMP777.

\section{References}

1. Bray F, Ferlay J, Laversanne M, Brewster DH, Gombe Mbalawa C, Kohler B, Pineros M, Steliarova-Foucher E, Swaminathan R, Antoni S, 
Soerjomataram I, Forman D: Cancer incidence in five continents: inclusion criteria, highlights from Volume $\mathrm{X}$ and the global status of cancer registration. Int J Cancer 2015, 137:2060-2071

2. Goldenring JR, Nomura S: Differentiation of the gastric mucosa III. Animal models of oxyntic atrophy and metaplasia. Am J Physiol Gastrointest Liver Physiol 2006, 291:G999-G1004

3. Goldenring JR, Nam KT: Oxyntic atrophy, metaplasia, and gastric cancer. Prog Mol Biol Transl Sci 2010, 96:117-131

4. Weis VG, Goldenring JR: Current understanding of SPEM and its standing in the preneoplastic process. Gastric Cancer 2009, 12: 189-197

5. Choi E, Hendley AM, Bailey JM, Leach SD, Goldenring JR: Expression of activated Ras in gastric chief cells of mice leads to the full spectrum of metaplastic lineage transitions. Gastroenterology 2016, 150:918-930.e13

6. Lee HJ, Nam KT, Park HS, Kim MA, Lafleur BJ, Aburatani H, Yang HK, Kim WH, Goldenring JR: Gene expression profiling of metaplastic lineages identifies $\mathrm{CDH} 17$ as a prognostic marker in early stage gastric cancer. Gastroenterology 2010, 139: 213-225.e3

7. Fox JG, Li X, Cahill RJ, Andrutis K, Rustgi AK, Odze R, Wang TC: Hypertrophic gastropathy in Helicobacter felis-infected wild-type C57BL/6 mice and p53 hemizygous transgenic mice. Gastroenterology 1996, 110:155-166

8. Nam KT, Lee HJ, Sousa JF, Weis VG, O'Neal RL, Finke PE, RomeroGallo J, Shi G, Mills JC, Peek RM Jr, Konieczny SF, Goldenring JR: Mature chief cells are cryptic progenitors for metaplasia in the stomach. Gastroenterology 2010, 139:2028-2037.e9

9. Segatto O, Anastasi S, Alema S: Regulation of epidermal growth factor receptor signalling by inducible feedback inhibitors. J Cell Sci 2011 , 124:1785-1793

10. Gur G, Rubin C, Katz M, Amit I, Citri A, Nilsson J, Amariglio N, Henriksson R, Rechavi G, Hedman H, Wides R, Yarden Y: LRIG1 restricts growth factor signaling by enhancing receptor ubiquitylation and degradation. EMBO J 2004, 23:3270-3281

11. Laederich $\mathrm{MB}$, Funes-Duran $\mathrm{M}$, Yen L, Ingalla $\mathrm{E}, \mathrm{Wu} \mathrm{X}$, Carraway KL 3rd, Sweeney C: The leucine-rich repeat protein LRIG1 is a negative regulator of ErbB family receptor tyrosine kinases. J Biol Chem 2004, 279:47050-47056

12. Jensen KB, Watt FM: Single-cell expression profiling of human epidermal stem and transit-amplifying cells: lrig1 is a regulator of stem cell quiescence. Proc Natl Acad Sci U S A 2006, 103: $11958-11963$

13. Jensen KB, Collins CA, Nascimento E, Tan DW, Frye M, Itami S, Watt FM: Lrig1 expression defines a distinct multipotent stem cell population in mammalian epidermis. Cell Stem Cell 2009, 4: 427-439

14. Powell AE, Wang Y, Li Y, Poulin EJ, Means AL, Washington MK, Higginbotham JN, Juchheim A, Prasad N, Levy SE, Guo Y, Shyr Y, Aronow BJ, Haigis KM, Franklin JL, Coffey RJ: The pan-ErbB negative regulator Lrig1 is an intestinal stem cell marker that functions as a tumor suppressor. Cell 2012, 149:146-158

15. Choi E, Lantz TL, Vlacich G, Keeley TM, Samuelson LC, Coffey RJ, Goldenring JR, Powell AE: Lrig1+ gastric isthmal progenitor cells restore normal gastric lineage cells during damage recovery in adult mouse stomach. Gut 2018, 67:1595-1605

16. Wong VW, Stange DE, Page ME, Buczacki S, Wabik A, Itami S, van de Wetering M, Poulsom R, Wright NA, Trotter MW, Watt FM, Winton DJ, Clevers H, Jensen KB: Lrig1 controls intestinal stem-cell homeostasis by negative regulation of ErbB signalling. Nat Cell Biol 2012, 14:401-408

17. Hedman H, Henriksson R: LRIG inhibitors of growth factor signalling - double-edged swords in human cancer? Eur J Cancer 2007, 43: 676-682

18. Ljuslinder I, Golovleva I, Palmqvist R, Oberg A, Stenling R, Jonsson Y, Hedman H, Henriksson R, Malmer B: LRIG1 expression in colorectal cancer. Acta Oncol 2007, 46:1118-1122
19. Miller JK, Shattuck DL, Ingalla EQ, Yen L, Borowsky AD, Young LJ, Cardiff RD, Carraway KL 3rd, Sweeney C: Suppression of the negative regulator LRIG1 contributes to ErbB2 overexpression in breast cancer. Cancer Res 2008, 68:8286-8294

20. Thomasson M, Hedman H, Guo D, Ljungberg B, Henriksson R: LRIG1 and epidermal growth factor receptor in renal cell carcinoma: a quantitative RT-PCR and immunohistochemical analysis. Br J Cancer 2003, 89:1285-1289

21. Barrett $\mathrm{T}$, Wilhite SE, Ledoux $\mathrm{P}$, Evangelista C, Kim IF, Tomashevsky M, Marshall KA, Phillippy KH, Sherman PM, Holko M, Yefanov A, Lee H, Zhang N, Robertson CL, Serova N, Davis S, Soboleva A: NCBI GEO: archive for functional genomics data sets—update. Nucleic Acids Res 2013, 41:D991-D995

22. Kalluri R, Weinberg RA: The basics of epithelial-mesenchymal transition. J Clin Invest 2009, 119:1420-1428

23. Szasz AM, Lanczky A, Nagy A, Forster S, Hark K, Green JE, Boussioutas A, Busuttil R, Szabo A, Gyorffy B: Cross-validation of survival associated biomarkers in gastric cancer using transcriptomic data of 1,065 patients. Oncotarget 2016, 7:49322-49333

24. Pisanu ME, Noto A, De Vitis C, Morrone S, Scognamiglio G, Botti G, Venuta F, Diso D, Jakopin Z, Padula F, Ricci A, Mariotta S, Giovagnoli MR, Giarnieri E, Amelio I, Agostini M, Melino G, Ciliberto G, Mancini R: Blockade of Stearoyl-CoA-desaturase 1 activity reverts resistance to cisplatin in lung cancer stem cells. Cancer Lett 2017, 406:93-104

25. Vries RG, Huch M, Clevers H: Stem cells and cancer of the stomach and intestine. Mol Oncol 2010, 4:373-384

26. Wang Y, Shi C, Lu Y, Poulin EJ, Franklin JL, Coffey RJ: Loss of Lrig1 leads to expansion of Brunner glands followed by duodenal adenomas with gastric metaplasia. Am J Pathol 2015, 185:1123-1134

27. Hoffmann W: Current status on stem cells and cancers of the gastric epithelium. Int J Mol Sci 2015, 16:19153-19169

28. Fox JG, Wang TC: Inflammation, atrophy, and gastric cancer. J Clin Invest 2007, 117:60-69

29. Correa P, Houghton J: Carcinogenesis of Helicobacter pylori. Gastroenterology 2007, 133:659-672

30. Lennerz JK, Kim SH, Oates EL, Huh WJ, Doherty JM, Tian X, Bredemeyer AJ, Goldenring JR, Lauwers GY, Shin YK, Mills JC: The transcription factor MIST1 is a novel human gastric chief cell marker whose expression is lost in metaplasia, dysplasia, and carcinoma. Am J Pathol 2010, 177:1514-1533

31. Goldenring JR, Nam KT, Wang TC, Mills JC, Wright NA: Spasmolytic polypeptide-expressing metaplasia and intestinal metaplasia: time for reevaluation of metaplasias and the origins of gastric cancer. Gastroenterology 2010, 138:2207-2210.e1

32. Nam KT, Lee HJ, Mok H, Romero-Gallo J, Crowe JE Jr, Peek RM Jr, Goldenring JR: Amphiregulin-deficient mice develop spasmolytic polypeptide expressing metaplasia and intestinal metaplasia. Gastroenterology 2009, 136:1288-1296

33. Yoshizawa N, Takenaka Y, Yamaguchi H, Tetsuya T, Tanaka H, Tatematsu M, Nomura S, Goldenring JR, Kaminishi M: Emergence of spasmolytic polypeptide-expressing metaplasia in Mongolian gerbils infected with Helicobacter pylori. Lab Invest 2007, 87:1265-1276

34. Watanabe T, Tada M, Nagai H, Sasaki S, Nakao M: Helicobacter pylori infection induces gastric cancer in Mongolian gerbils. Gastroenterology 1998, 115:642-648

35. Honda S, Fujioka T, Tokieda M, Satoh R, Nishizono A, Nasu M: Development of Helicobacter pylori-induced gastric carcinoma in Mongolian gerbils. Cancer Res 1998, 58:4255-4259

36. Petersen CP, Weis VG, Nam KT, Sousa JF, Fingleton B, Goldenring JR: Macrophages promote progression of spasmolytic polypeptide-expressing metaplasia after acute loss of parietal cells. Gastroenterology 2014, 146:1727-1738.e8

37. Weis VG, Sousa JF, LaFleur BJ, Nam KT, Weis JA, Finke PE, Ameen NA, Fox JG, Goldenring JR: Heterogeneity in mouse spasmolytic polypeptide-expressing metaplasia lineages identifies markers of metaplastic progression. Gut 2013, 62:1270-1279 
38. Petersen CP, Meyer AR, De Salvo C, Choi E, Schlegel C, Petersen A, Engevik AC, Prasad N, Levy SE, Peebles RS, Pizarro TT, Goldenring JR: A signalling cascade of IL-33 to IL-13 regulates metaplasia in the mouse stomach. Gut 2018, 67:805-817

39. Mills JC, Goldenring JR: Metaplasia in the stomach arises from gastric chief cells. Cell Mol Gastroenterol Hepatol 2017, 4: $85-88$

40. Goldenring JR: Pyloric metaplasia, pseudopyloric metaplasia, ulcerassociated cell lineage and spasmolytic polypeptide-expressing metaplasia: reparative lineages in the gastrointestinal mucosa. J Pathol 2018, 245:132-137

41. Wang Y, Poulin EJ, Coffey RJ: LRIG1 is a triple threat: ERBB negative regulator, intestinal stem cell marker and tumour suppressor. Br J Cancer 2013, 108:1765-1770

42. Goldoni S, Iozzo RA, Kay P, Campbell S, McQuillan A, Agnew C, Zhu JX, Keene DR, Reed CC, Iozzo RV: A soluble ectodomain of LRIG1 inhibits cancer cell growth by attenuating basal and liganddependent EGFR activity. Oncogene 2007, 26:368-381

43. Kou C, Zhou T, Han X, Zhuang H, Qian H: LRIG1, a 3p tumor suppressor, represses EGFR signaling and is a novel epigenetic silenced gene in colorectal cancer. Biochem Biophys Res Commun 2015, 464:519-525
44. Zhou L, Li X, Zhou F, Jin Z, Chen D, Wang P, Zhang S, Zhuge Y, Shang Y, Zou X: Downregulation of leucine-rich repeats and immunoglobulin-like domains 1 by microRNA-20a modulates gastric cancer multidrug resistance. Cancer Sci 2018, 109:1044-1054

45. Shao LM, Yang JA, Wang YF, Wu P, Li JQ, Chen QX: MicroRNA19a promotes glioma cell growth by repressing LRIG1. Int J Clin Exp Med 2014, 7:5067-5074

46. Jeon TY, Han ME, Lee YW, Lee YS, Kim GH, Song GA, Hur GY, Kim JY, Kim HJ, Yoon S, Baek SY, Kim BS, Kim JB, Oh SO: Overexpression of stathmin 1 in the diffuse type of gastric cancer and its roles in proliferation and migration of gastric cancer cells. Br J Cancer 2010, 102:710-718

47. Ko YS, Cho SJ, Park J, Kim Y, Choi YJ, Pyo JS, Jang BG, Park JW, Kim WH, Lee BL: Loss of FOXO1 promotes gastric tumour growth and metastasis through upregulation of human epidermal growth factor receptor 2/neu expression. Br J Cancer 2015, 113:1186-1196

48. Gunasinghe NP, Wells A, Thompson EW, Hugo HJ: Mesenchymalepithelial transition (MET) as a mechanism for metastatic colonisation in breast cancer. Cancer Metastasis Rev 2012, 31:469-478

49. Creighton CJ, Chang JC, Rosen JM: Epithelial-mesenchymal transition (EMT) in tumor-initiating cells and its clinical implications in breast cancer. J Mammary Gland Biol Neoplasia 2010, 15:253-260 